\title{
Microarray analysis reveals differentially expressed IncRNAs in benign epithelial ovarian cysts and normal ovaries
}

\author{
XIAOGUANG LIU ${ }^{1,2^{*}}$, CHENCHENG DAI ${ }^{1,3^{*}}$, GENMEI JIA ${ }^{1,3}$, SUJUAN XU $^{4}$, ZIYI FU ${ }^{1}$, \\ JUAN XU ${ }^{1,2}$, QING LI $^{5}$, HONGJIE RUAN ${ }^{2}$ and PENGFEI XU ${ }^{1}$
}

\author{
${ }^{1}$ Nanjing Maternal and Child Health Institute, and ${ }^{2}$ Department of Gynecology, Nanjing Maternal and \\ Child Health Care Hospital, Obstetrics and Gynecology Hospital Affiliated to Nanjing Medical University, \\ Nanjing, Jiangsu 210004; ${ }^{3}$ The First Clinical Medical College of Nanjing Medical University, Nanjing, Jiangsu 210029; \\ ${ }^{4}$ Department of Clinical Laboratory, Nanjing Maternal and Child Health Care Hospital, Obstetrics and Gynecology Hospital \\ Affiliated to Nanjing Medical University, Nanjing, Jiangsu 210004; ${ }^{5}$ Department of Pathology, \\ Shanghai Pudong New Area People's Hospital, Shanghai 201040, P.R. China
}

Received December 28, 2016; Accepted June 9, 2017

DOI: 10.3892/or.2017.5741

\begin{abstract}
Recent studies suggest that long non-coding RNAs (lncRNAs) play crucial roles in many types of human malignant cancers. However, the function of lncRNAs in benign tumors remains poorly understood. In the present study, to explored the potential roles of IncRNAs in benign epithelial ovarian cysts (BEOCs) which commonly occur in young women and possess malignant potential, we described the expression profile of the lncRNAs between BEOC and normal ovarian tissues using lncRNA microarray techniques. The results showed that 1,325 transcripts of IncRNAs (1,014 upregulated and 311 downregulated) were differentially expressed in the BEOCs compared with the normal controls [absolute fold-change $\geq 2$, false discovery rate (FDR) $<0.05$ ]. We also conducted quantitative real-time PCR (qPCR) to confirm the microarray data. The results of qPCR revealed that the expression trend of 6 randomly selected lncRNAs was consistent with the microarray data. Furthermore, candidate IncRNAs were characterized by pathway analysis and Gene Ontology (GO). The present study is the first to demonstrate different expression profiles of lncRNAs between BEOCs and normal
\end{abstract}

Correspondence to: $\mathrm{Dr}$ Pengfei $\mathrm{Xu}$, Nanjing Maternal and Child Health Institute, Nanjing Maternal and Child Health Care Hospital, Obstetrics and Gynecology Hospital Affiliated to Nanjing Medical University, 123 Tianfeixiang, Mochou Road, Nanjing, Jiangsu 210004, P.R. China

E-mail: pengfeixu@njmu.edu.cn

Dr Hongjie Ruan, Department of Gynecology, Nanjing Maternal and Child Health Care Hospital, Obstetrics and Gynecology Hospital Affiliated to Nanjing Medical University, 123 Tianfeixiang, Mochou Road, Nanjing, Jiangsu 210004, P.R. China

E-mail: hongjie_ruan@126.com

*Contributed equally

Key words: IncRNA, benign, ovarian cyst, microarray, qPCR ovarian tissues. These lncRNAs may play a crucial role in the pathological process of BEOCs.

\section{Introduction}

Ovarian tumors are the leading cause of death among all gynecologic tumors (1). In the US, 22,280 cases of ovarian cancer were estimated to be diagnosed and it was estimated that 14,240 women died from ovarian cancer in 2016 (2). Despite modern surgical techniques and chemotherapy, the prognosis of ovarian cancer remains poor (3). Ovarian tumors are a common type of neoplasm in women and the histological type is various and complex. Epithelial ovarian tumors are the most common type of ovarian tumor and represents $50-70 \%$ of all primary ovarian tumors. According to the characteristics of the tumor cells and the severity to health, epithelial ovarian tumors can be divided into three types: benign, borderline and malignant ovarian tumors. In various cases, benign tumors can develop into a malignant tumor (4-7), which suggests that benign ovarian tumors have an increased risk to transform into malignant tumors due to the changes of various genes (8) or proteins (9). However, extensive research has mainly focused on malignant epithelial ovarian cancer, and benign ovarian tumors have not been a principal focus of research. In fact, there are far more benign lesions occurring in the epithelial ovary, and these are commonly diagnosed during pregnancy (10). In addition, these are also associated with an increased risk of malignant epithelial ovarian cancer. Therefore, a deeper understanding of benign epithelial ovarian cysts (BEOCs) may not only provide more effective treatments for BEOCs, but may also reduce the incidence of malignant epithelial ovarian cancer.

Long non-coding RNAs (lncRNAs) are a type of RNAs that are longer than 200 nucleotides in length and without coding protein capacity (11). IncRNAs have been previously considered as 'transcriptional noise' for a long time (12). Recently, studies have confirmed that lncRNAs play a critical role in the development of cancer, including ovarian cancer. Emerging 
evidence suggests that IncRNAs are associated with ovarian cancer biological behaviors such as cell proliferation $(13,14)$, apoptosis $(15,16)$, and invasion and metastasis $(17,18)$. Moreover, due to tissue-specificity, some lncRNAs may served as potential biomarkers for cancer prognosis, including ovarian cancer (19). However, these studies have only focused on the regulation of lncRNAs in malignant ovarian cancer, and little research has been carried out on the relationship between lncRNAs and benign ovarian tumors. Therefore, lncRNA expression profiles in BEOCs may help us to better understand BEOC pathogenesis.

In the present study, we described the distinct expression profiles of lncRNAs in BEOC and normal ovarian epithelial tissues. In total, 1,014 transcripts of lncRNAs were upregulated and 311 transcripts of lncRNAs were downregulated in BEOCs compared with normal controls [absolute fold-change $\geq 2$, false discovery rate $(\mathrm{FDR})<0.05]$. Moreover, we also examined the Gene Ontology (GO) enrichment of their associated proteincoding genes and performed pathway analyses to analyze the potential function of these differentially expressed lncRNAs. The present study may aid in elucidating the tumorigenesis of ovarian epithelial tissue and decrease the incidence of malignant transformation in regards to BEOCs.

\section{Materials and methods}

Tissue collection. Samples of BEOCs and normal ovarian tissues were collected from the Gynecologic Oncology Department of Nanjing Maternal and Child Health Hospital (Nanjing, China) from 2014 to 2016. No patient received chemotherapy or radiotherapy before surgery. Informed consent for the use of the tissues was obtained from all patients. Finally, 10 cases of normal ovarian tissues and 17 cases of BEOCs were collected and immediately stored in RNAsafety ${ }^{\mathrm{TM}}$ and frozen at $-80^{\circ} \mathrm{C}$ before the experiments. All the tissues were confirmed through histopathological diagnoses. The present study was approved by the Ethics Review Committee of Nanjing Maternity and Child Health Care Hospital.

Total RNA extraction. Frozen tissues were dissolved in TRIzol reagent (Life Technologies, Grand Island, NY, USA). Total RNA was extracted according to the manufacturer's protocol (Invitrogen, Carlsbad, CA, USA). NanoDrop and the Agilent 2100 Bioanalyzer (Agilent Technologies, Inc., Santa Clara, CA, USA) were used to check the quantification and quality of the extracted RNA, respectively. The extracted RNA samples were stored at $-80^{\circ} \mathrm{C}$ for further experiments. Complementary DNA (cDNA) was synthesized from $1 \mu \mathrm{g}$ of total-RNA using a PrimesScript ${ }^{\mathrm{TM}}$ RT Master Mix kit (Applied Takara, Dalian, China) with random hexamer primers in a final volume of $20 \mathrm{ml}$. The condition of reverse-transcription reaction was as follows: $37^{\circ} \mathrm{C}$ for $15 \mathrm{~min}, 85^{\circ} \mathrm{C}$ for $5 \mathrm{sec}$ and $4^{\circ} \mathrm{C}$ for $10 \mathrm{~min}$.

Microarray analysis. To screen the global profiling of human lncRNAs and protein-coding transcripts, we profiled three BEOC tissues and three normal ovarian epithelial tissues with ArrayStar Human Microarray V3.0. The lncRNAs were searched for using authoritative databases such as RefSeq, Ensembl and UCSC Known Genes and related studies while the mRNAs were collected from RefSeq and GENCODE. To recognize every individual transcript exactly, each transcript was described with a specific exon or splice junction probe. Both positive probes (the housekeeping genes) and negative probes were printed onto the array for hybridization quality control. The sample processing and microarray hybridization were performed in terms of the Agilent One-Color MicroarrayBased Gene Expression Analysis protocol (Agilent Technology). Briefly, $1 \mathrm{mg}$ of total RNA was obtained for purification by removing the rRNA (mRNA-ONLY Eukaryotic mRNA Isolation kit; Epicentre, Madison, WI, USA). Then, each sample was amplified and transcribed into fluorescent cRNA along the entire length of the transcripts without $3^{\prime}$ bias utilizing a random priming method. Agilent Quick Amp Labeling kit was employed to normalize the values, and then, lncRNAs and mRNAs, for which at least one out of two groups had flags in present or marginal, were chosen for further data analysis. Additionally, hierarchical clustering and combined analyses were performed using homemade scripts.

Quantitative real-time PCR ( $q P C R$ ). qPCR was performed to detect the relative gene expression using Power SYBR-Green PCR Master Mix (2X Applied Biosystems) according to the standard protocol. GAPDH was taken as an internal reference. The qPCR reaction conditions were set as follows: an initial denaturation at $95^{\circ} \mathrm{C}$ for $30 \mathrm{sec}$, followed by 40 PCR cycles at $95^{\circ} \mathrm{C}$ for $5 \mathrm{sec}$ and $60^{\circ} \mathrm{C}$ for $34 \mathrm{sec}$. Finally annealing and extension at $95^{\circ} \mathrm{C}$ for $15 \mathrm{sec}, 60^{\circ} \mathrm{C}$ for $60 \mathrm{sec}$ and $95^{\circ} \mathrm{C}$ for $15 \mathrm{sec}$. Each sample was detected in triplicates. The fold-change was calculated with the $\triangle \mathrm{CT}$ method to describe the relative gene expression in BEOC samples relative to the normal ovarian tissue samples. All of the primers are presented in Table I.

GO and pathway analyses. Pathway and GO analyses were applied to determine the potential roles of differentially expressed lncRNAs in biological pathways or GO terms. The predicted target genes of the differentially expressed lncRNAs were mapped to GO terms in the Database for Annotation Visualization and Integrated Discovery (DAVID) (http://david.abcc.ncifcrf.gov/). Fisher's exact test was used to ascertain whether true differences existed between the groups. In addition, we used the Kyoto Encyclopedia of Genes and Genomes (KEGG) (http://www.kegg.jp/) to confirm the pathway enrichment analysis. The ontology covers three domains: biological process (BP), cellular component (CC) and molecular function (MF). The threshold of significance was defined by FDR.

Statistical analysis. Differential expression levels of lncRNAs were selected by fold-change filtering (absolute fold-change $>2.0$ ). Independent samples t-test between two groups was used, and Fisher's exact test was used in GO and pathway analyses. A value of FDR $<0.05$ was considered statistically significant. Computer-based calculations were conducted using SPSS version 20.0 (SPSS, Inc., Chicago, IL, USA).

\section{Results}

Differentially expressed lncRNAs and mRNAs in BEOCs compared with normal ovarian tissues. Firstly, to explore the 
Table I. qPCR primers used in the present study.

\begin{tabular}{lll}
\hline lncRNAs & \multicolumn{1}{c}{ Forward (5'-3') } & \multicolumn{1}{c}{ Reverse $\left(5^{\prime}-3^{\prime}\right)$} \\
\hline LOC339166 & GCCTCTCTGGAGCTGAATCG & CGTGCCAGTGGGTTTCCTAA \\
LOC440214 & CCACCCCAAAGAAGATGCTG & ACAGGAGACAAAGCCTTCGC \\
LOC644656 & AATTAGTTGTGGCCGTTGCG & ATCTTTAGTCGGCCTGGTGC \\
NENF & CAGGAGCAGGTTCTTGGGAG & CCAAGGACAACAGGAGGCAT \\
RP11-471J12.1 & TCAGCCCACCTGCTCCAA & TGATCTGTGCCTTCCTGGTACA \\
MEG3 & CTGCCCATCTACACCTCACG & CTCTCCGCCGTCTGCGCTAGGGGCT \\
GAPDH & CCAGAACATCATCCCTGCCT & CCTGCTTCACCACCTTCTTG
\end{tabular}

A

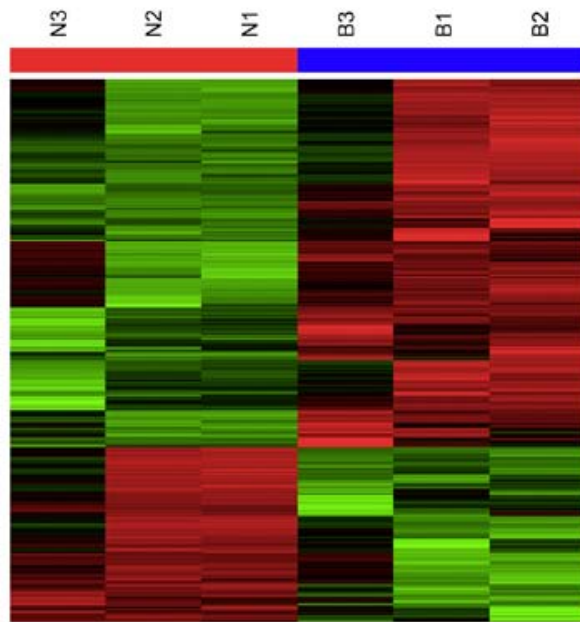

c

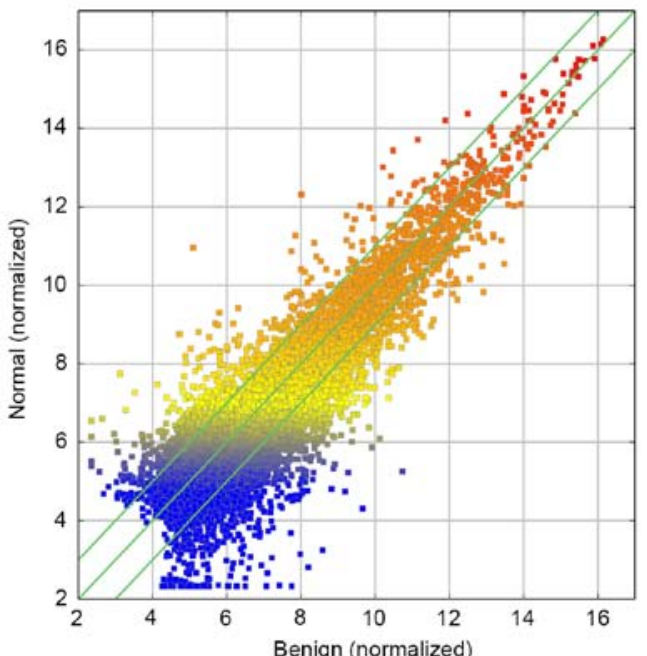

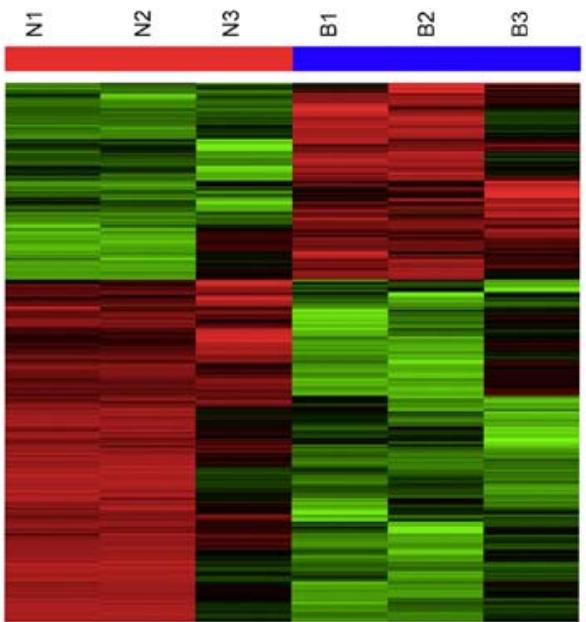

D

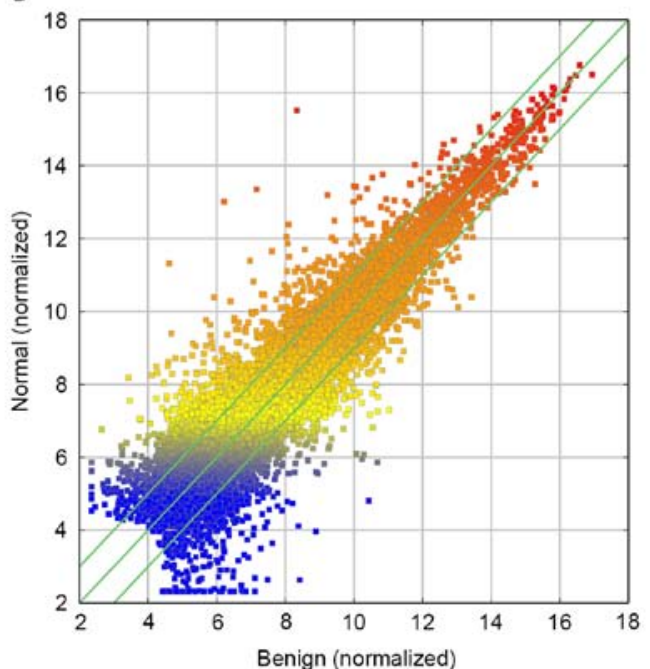

Figure 1. Differentially expressed lncRNAs in BEOCs compared with normal ovarian tissues. (A and B) Differentially expressed lncRNAs and mRNAs between BEOCs and normal ovarian tissues were analyzed using hierarchical clustering; 'red' indicates high relative expression, and 'green' indicates low relative expression. (C and D) Scatter plots were used to assess expression variations of lncRNAs and mRNAs between BEOCs and normal ovaries. IncRNAs and mRNAs above the top green line and below the green line exhibit a $>2.0$ fold-change.

altered lncRNAs in the BEOCs, we determined the lncRNA and mRNA expression profiles using microarray analyses of normal ovarian and BEOC tissues. Heatmaps and scatter-plots were used to describe the variation in lncRNA expression among normal ovarian, benign ovarian cysts and malignant epithelial ovarian cancer tissues (Fig. 1). All lncRNAs and mRNAs with a signal altered by 2 -fold and with a false discovery rate (FDR) $<0.05$ were identified as statistically altered. Finally, 1,325 transcripts of lncRNAs (1,014 upregulated and 311 downregulated) and 1,563 mRNAs (613 upregulated and 950 downregulated) were found to be differentially expressed in BEOC tissues compared with normal controls. Lists of the top 20 upregulated and downregulated lncRNAs identified in the microarray analyses are presented in Tables II and III. 


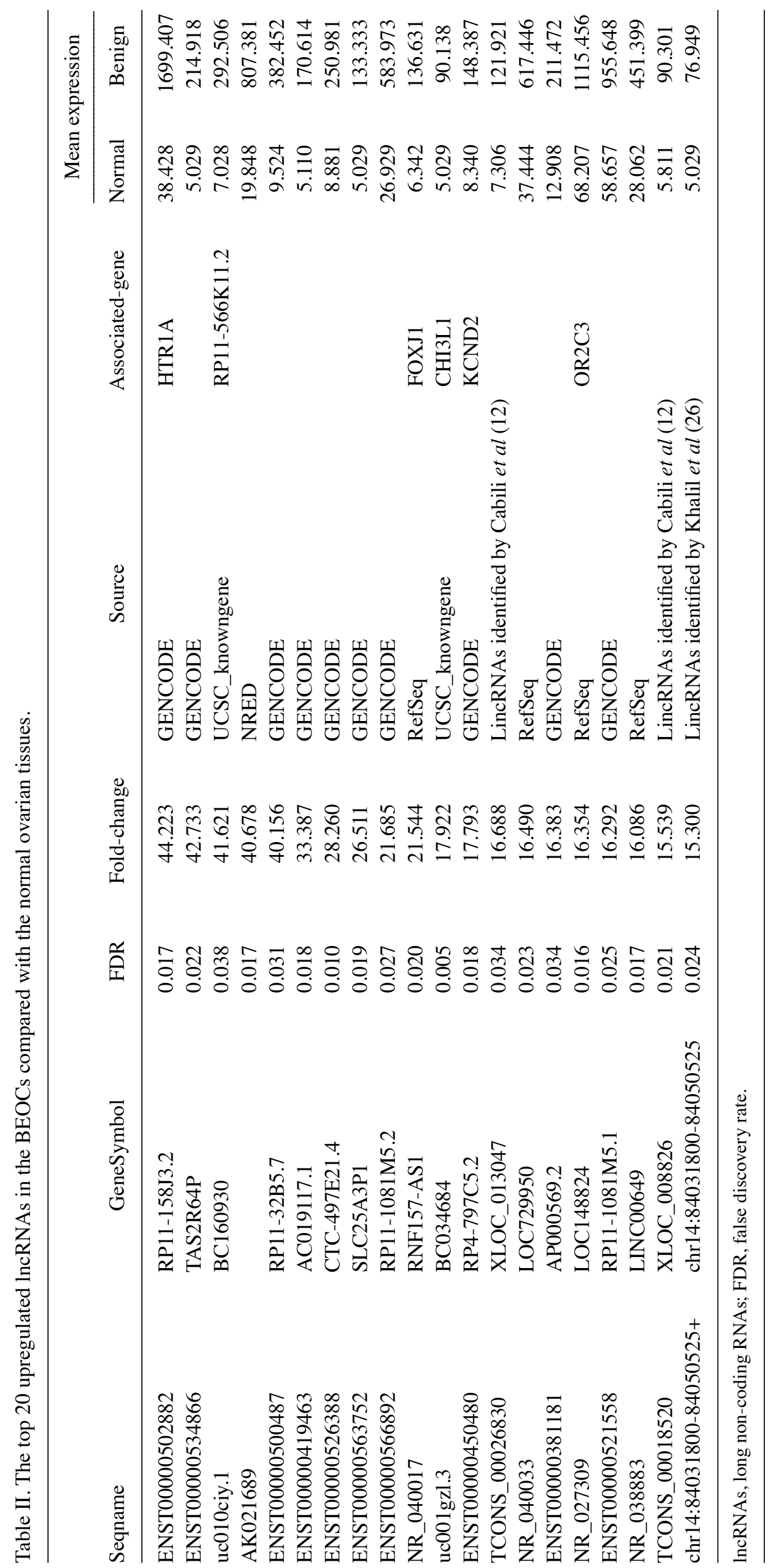




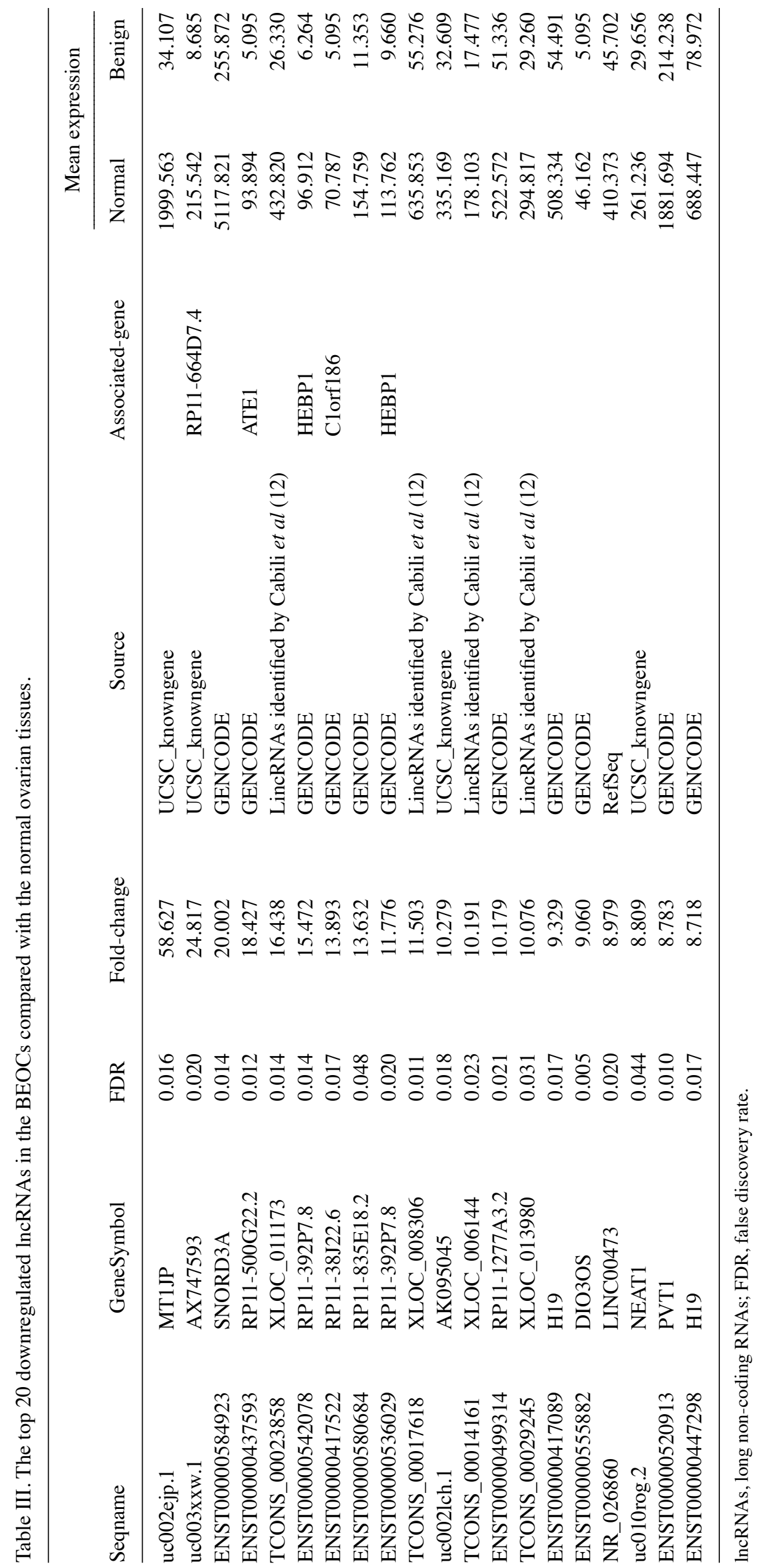



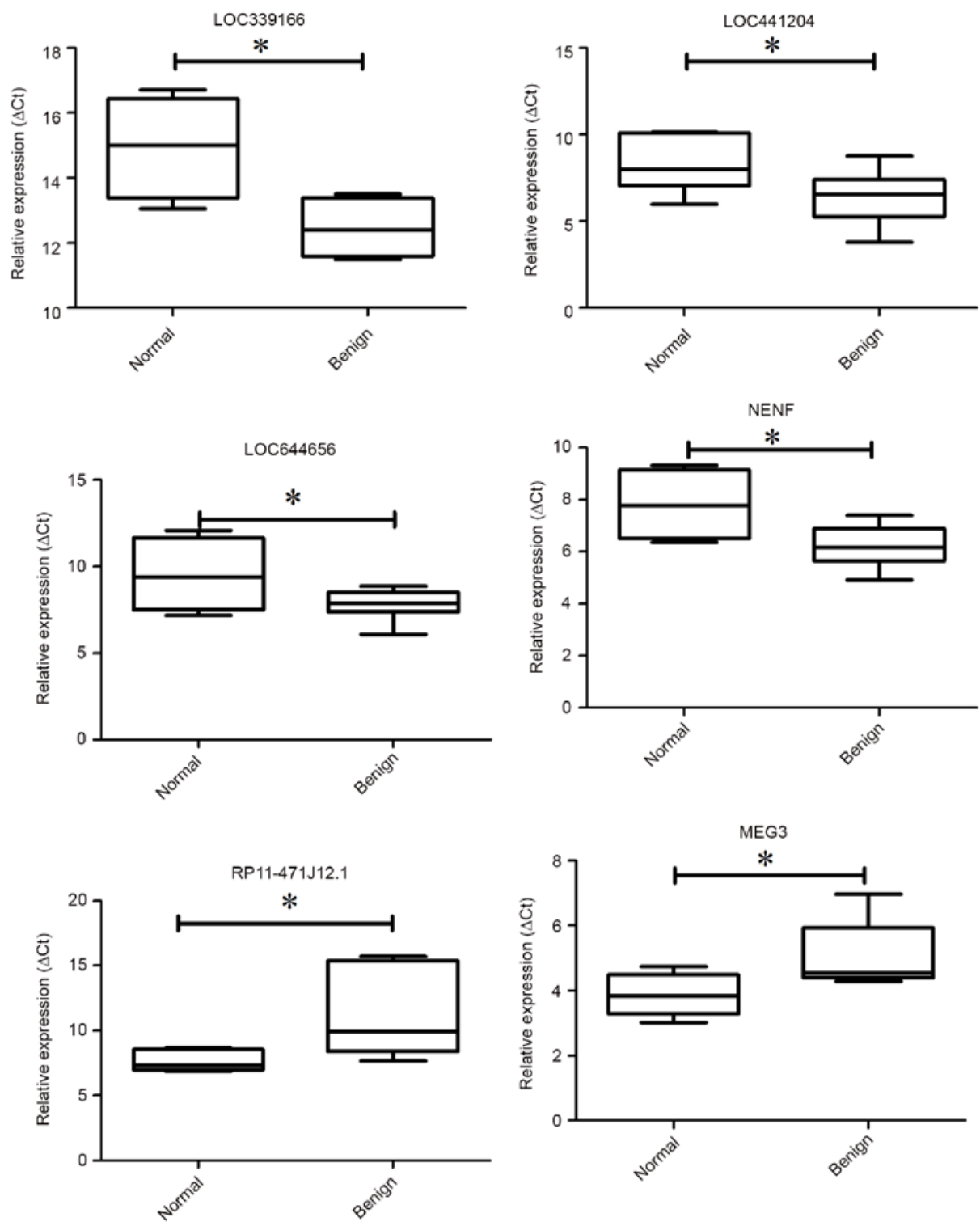

Figure 2. Validation by qPCR of candidate lncRNAs in BEOCs and normal ovarian tissues. The relative expression levels of candidate lncRNAs were detected by qPCR in 8 normal ovarian, 17 benign cyst and 15 malignant EOC samples. The $\Delta \mathrm{Ct}$ values of the lncRNAs were determined by subtracting the GAPDH $\Delta C \mathrm{Ct}$ value. A smaller $\Delta \mathrm{Ct}$ value indicates higher expression levels. Data are presented as the relative expression level in tumor tissues (shown as $\Delta \mathrm{Ct}$ ); all " $\mathrm{P}<0.05$.

Validation of candidate lncRNAs by $q P C R$. To confirm the validity of the microarray data, we next conducted qPCR to detect the expression of the IncRNAs. We randomly selected 6 differentially expressed lncRNAs. Among the 6 lncRNAs, IncRNAs LOC339166, LOC441204, LOC644656 and NENF were upregulated whereas 1ncRNAs RP11-471J12.1 and MEG3 were downregulated in the BEOCs compared with the normal controls (Fig. 2). The result of qPCR confirmed that the expression trend of the 6 selected lncRNAs was consistent with the microarray data.

GO and pathway analyses of the differentially expressed lncRNAs. To investigate the function of altered lncRNAs in the BEOCs, we performed GO analysis which covered the following three domains: biological processes (BP), cellular components (CC) and molecular functions (MF). We found that the highest GO classifications targeted by the upregulated lncRNAs were single-organism process (Fig. 3A), membrane (Fig. 3B) and signal transducer activity (Fig. 3C). However, the highest GO classifications targeted by downregulated lncRNAs were cellular process (Fig. 3D), cell part (Fig. 3E) and binding, particularly protein binding (Fig. 3F). To map these lncRNAs to pathways, we also performed pathway analysis. The result indicated that the 9 main pathways corresponding to the upregulated transcripts and the most enriched network was "neuroactive ligand-receptor interaction' (Fig. 4A). The 9 main pathways are shown: i) neuroactive ligand-receptor interaction; ii) axon guidance; iii) mucin type O-glycan biosynthesis; iv) Staphylococcus areus infection; v) transcriptional misregulation in cancer; vi) GABAergic synapse; vii) carbohydrate digestion and absorption; viii) serotonergic synapse; ix) glutamatergic synapse. We also observed 10 main pathways corresponding to the downregulated transcripts (Fig. 4B): i) focal adhesion; ii) bacterial invasion of epithelial cells; iii) phagosome; iv) leukocyte transendothelial migration; 


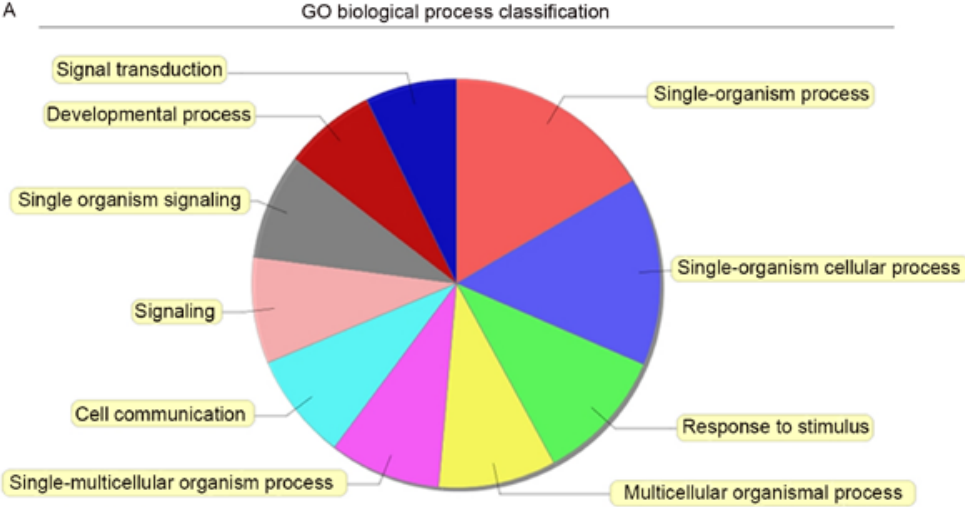

B

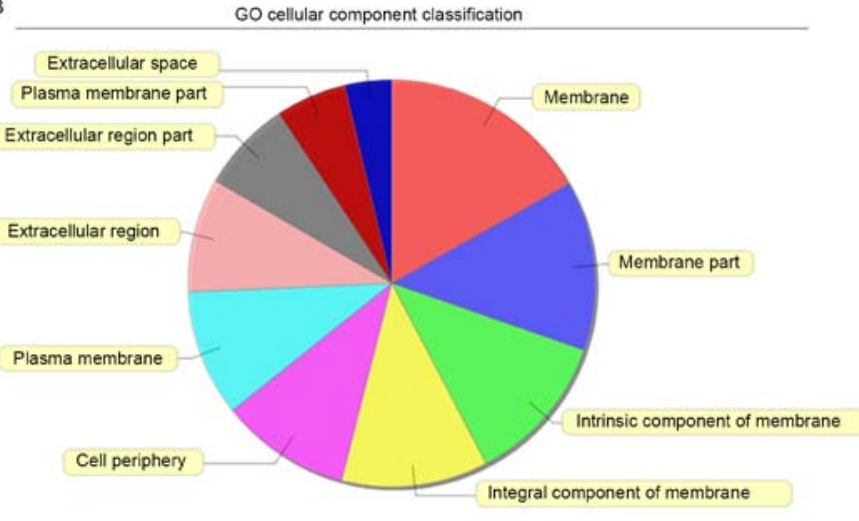

C GO molecular function classification

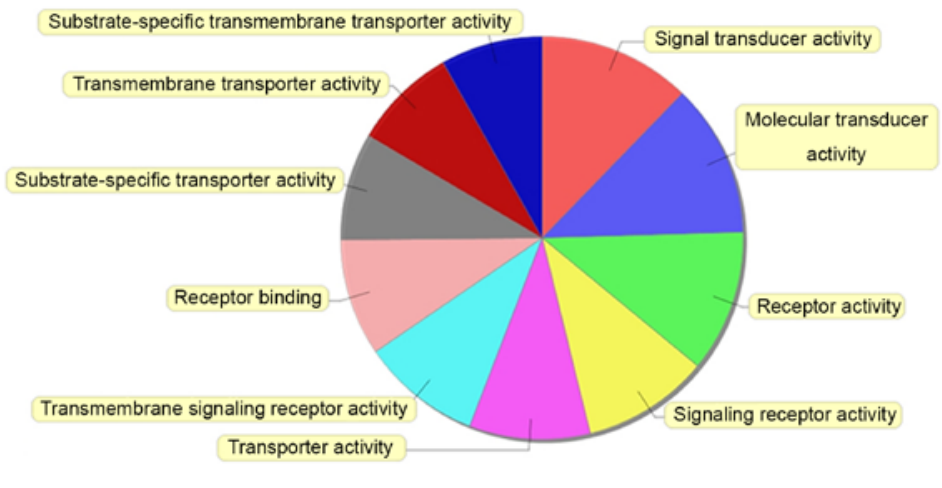

D GO biological process classification

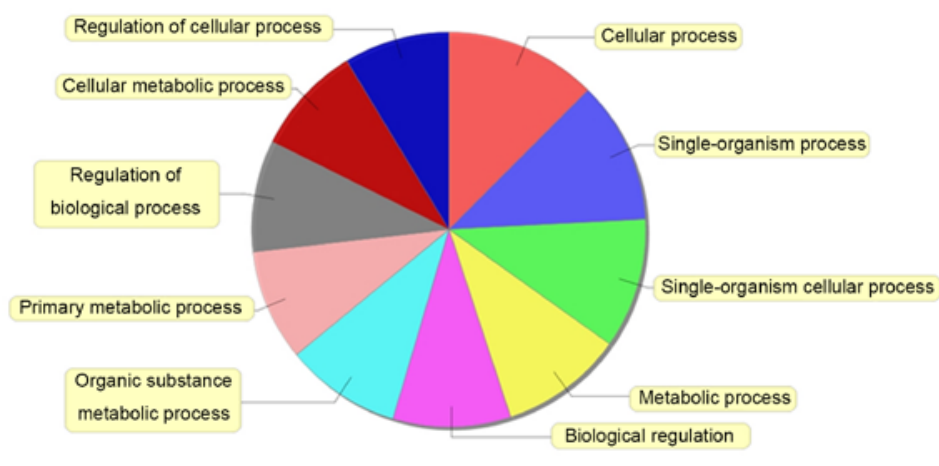

Figure 3. GO analysis of the differentially expressed lncRNAs. GO analysis results show the differentially expressed lncRNAs associated with biological processes (BP), cellular components (CC) and molecular functions (MF). The most frequent fold enrichment BP associated with (A) upregulated lncRNAs and (D) downregulated lncRNAs in BEOCs. The most frequent fold enrichment CC for (B) lncRNAs upregulated in BEOCs compared with normal control. The top $10 \mathrm{GO}$ terms of MF associated with (C) upregulated lncRNAs. 

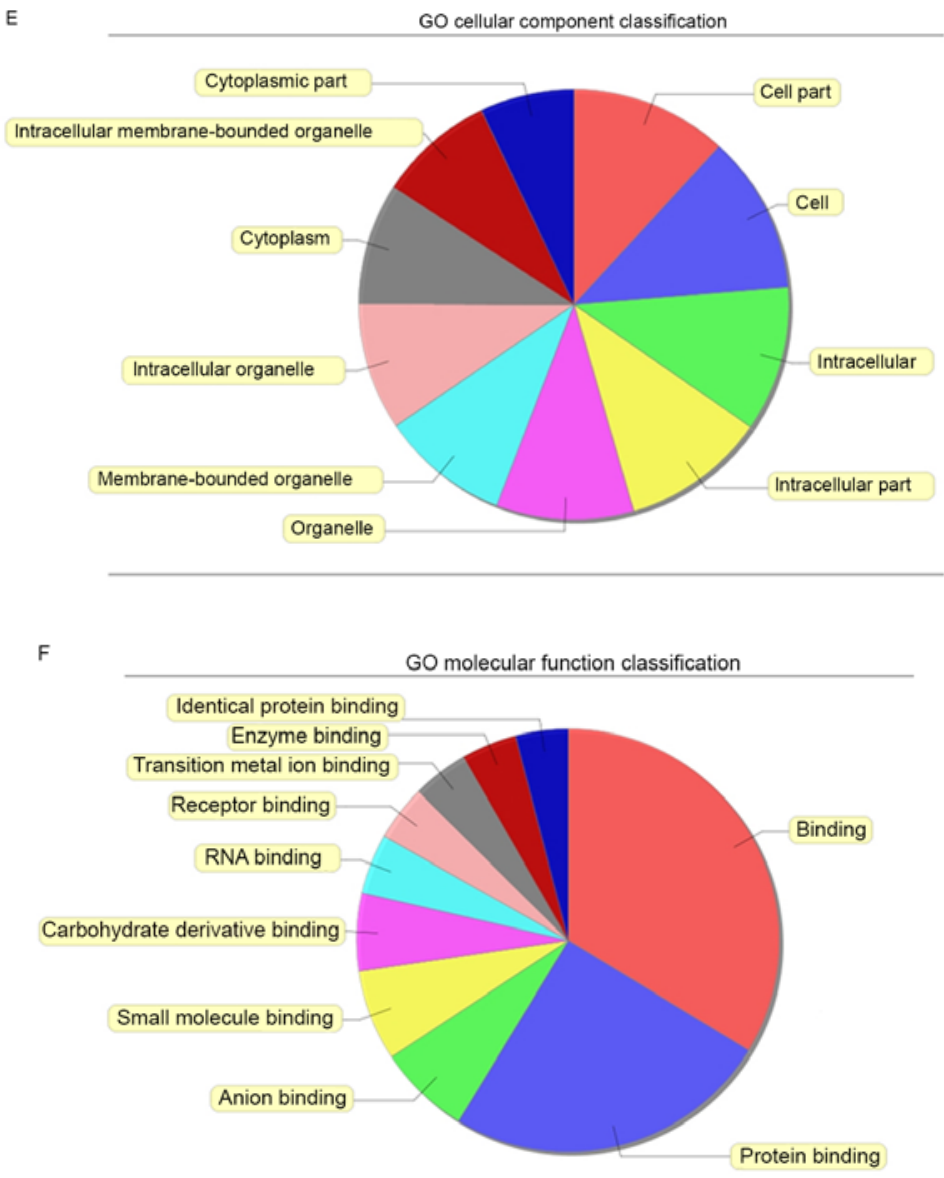

Figure 3. Continued. The most frequent fold enrichment for CC for (E) downregulated lncRNAs in BEOCs compared with normal control. The top $10 \mathrm{GO}$ terms of MF associated with (F) downregulated lncRNAs.
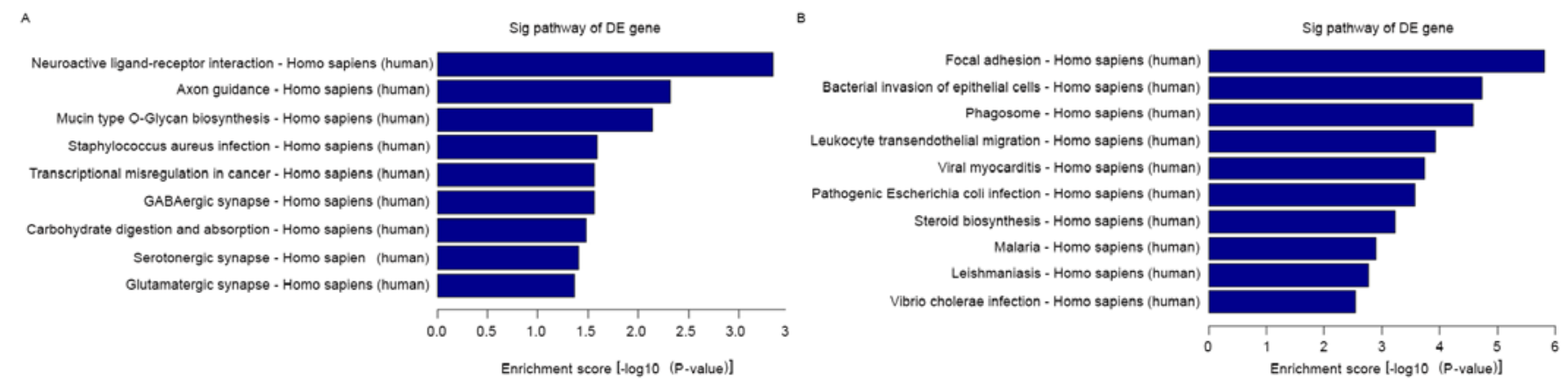

Figure 4. Pathway analysis of the differentially expressed lncRNAs. (A) The most frequent enrichment pathways corresponding to the lncRNAs upregulated in BEOC compared with normal tissues. (B) Top 10 pathways corresponding to the IncRNAs downregulated in BEOC tissues.

v) viral myocarditis; vi) pathogenic Escherichia coli infection; vii) steroid biosynthesis; viii) malaria; ix) leishmaniasis; x) Vibrio cholera infection. The most enriched network was 'focal adhesion' with 37 transcripts annotated with this term (Fisher P-value $=1.53367 \mathrm{E}-06$ ).

\section{Discussion}

BEOCs are the most common form of ovarian tumors in women, accounting for $\sim 80 \%$ of all ovarian masses (20). However, the molecular mechanisms related to the tumorigenesis of ovarian epithelial cells remains largely unknown. Increasing studies have claimed that IncRNAs are highly functional and have a crucial role in malignat ovarian cancer $(13,17,18,21,22)$. In our previous study, we identified dysregulation of many lncRNAs in malignant ovarian cancer compared with benign ovarian cysts and normal ovary. However, we also found that several lncRNAs, such as LEMD1-AS1 and AK 125532 were differentially expressed in BEOCs compared with these in the normal control (23). Thus, we next identified differentially expressed lncRNAs in BEOCs compared with normal ovarian tissues. To the best of our knowledge, the present study is the 
first to investigate the lncRNA expression profiling in BEOCs compared with normal ovarian tissues. The present study provides a better understanding of the molecular mechanisms of BEOCs.

On the basis of the GO analysis, we found that the BP of upregulated and downregulated lncRNAs was both tightly associated with single-organism process and single-organism cellular process. These GO terms are also associated with death and cell proliferation (24). The destiny of BEOCs is critically regulated by the cell cycle and apoptosis process in which lncRNAs may play an important role. For CC, the top GO term of the upregulated lncRNAs was membrane part and in downregulated lncRNAs this was cell part. These results indicate that IncRNAs may primarily regulate various mRNAs which are located in the cell or on the membrane and exercise their function. It can also be observed that the highest frequency of the MF GO terms in the upregulated lncRNAs was molecular transducer activity. Many studies have reported that some lncRNAs act as a molecular transducer. For example, lncRNA-TUG1 is overexpressed in non-small cell lung cancer, and can be regulated by p53 and affect cell proliferation through HOXB7 expression (25). However, the top MF GO term in the downregulated lncRNAs was binding, particularly protein binding. Increasing studies suggest that the primary function of lncRNAs is the epigenetic regulation of coding gene through proteins or microRNAs. Many lncRNAs have been reported to recruit and bind to PRC2 (26) or other chromatin-associated proteins (27). In addition, accumulating evidence indicates that lncRNAs act as competing endogenous RNAs (ceRNAs) by 'sponging' microRNAs $(22,28,29)$.

In addition, pathway analysis displayed that the upregulated lncRNAs were mainly correlated with neuroactive ligand-receptor interaction and axon guidance. Various studies have reported that the sympathetic nervous system is important in the tumor microenvironment $(30,31)$, and autonomic nerve development promotes cancer progression (32). Therefore, we speculated that there may be a relationship between ovarian tumorigenesis and the dysregulation of neural signaling pathways. In contrast, the downregulated lncRNAs were mainly correlated with focal adhesion. Focal adhesion is important between two cells or between a cell and the extracellular matrix. Various coding genes such as E-cadherin play critical roles in focal adhesion and tumor metastasis when malignant potential is increased in ovarian cancer cells. Moreover, some lncRNAs such as H19 (33), HOTAIR and MALAT1 (34) were found to promote cancer aggression by regulating E-cadherin.

Research has shown that the expression of $\mathrm{H} 19$ is upregulated in many types of cancers including ovarian cancer. The ectopic expression of $\mathrm{H} 19$ was found to promote cancer cell proliferation and invasion, suggesting that $\mathrm{H} 19$ may function as an oncogene. In our microarray, the transcript of H19 (ENST00000422826) was overexpressed $\sim 3$-fold in BEOCs compared with that in normal tissues. The results also indicated that BEOCs may have malignancy potential. However, the transcripts of H19 (ENST00000417089, ENST00000447298, uc001lva.4, uc021qbz.1, ENST00000442037 and ENST00000446406) were reduced in the BEOCs. The results of PVT1 were also the same as H19; different transcripts had differential expression in the BEOCs. The different transcripts of lncRNAs had differential expression and function. However, confirmation and elucidation of this finding require further study.

Due to the important roles of lncRNAs in cancer, a growing number of studies have focused on the potential lncRNA-related biomarkers for tumors. 1ncRNA-related biomarkers aid clinicians to make accurate diagnoses and treatment decisions. Crea et al reported that PCAT18, the IncNRA which is specifically expressed in the prostate, could be a potential therapeutic target and biomarker for metastatic prostate cancer (35). In addition, similar lncRNA-related biomarkers with prognostic value have also been identified in other types of cancers such as breast (36), pancreatic (37) and lung cancer $(38,39)$. In ovarian cancer, various lncRNAs have been shown to have potential as prognostic biomarkers, such as HOTAIR (18), H19 (40) and HOXA11AS (41). However, lncRNA-related biomarkers in benign tumors still have not been reported. The present study may aid in the identification of lncRNA-related biomarkers for benign ovarian cysts.

In summary, we profiled the differential expression of lncRNAs and mRNAs between BEOCs and normal ovarian tissues. In total, 1,325 transcripts of lncRNAs and 1,563 mRNAs were found to be differentially expressed between BEOCs and normal ovarian control tissues (absolute fold-change $\geq 2$, FDR <0.05). Furthermore, dysregulated lncRNAs were characterized by a comprehensive examination of GO enrichment and pathway analysis by their associated protein-coding genes. Collectively, our findings suggest that lncRNAs play a critical role in the pathological process of BEOCs.

\section{Acknowledgements}

The present study was supported by the National Natural Science Foundation of China (grant no. 81402139).

\section{References}

1. Holschneider CH and Berek JS: Ovarian cancer: Epidemiology, biology, and prognostic factors. Semin Surg Oncol 19: 3-10, 2000.

2. Siegel RL, Miller KD and Jemal A: Cancer statistics, 2016. CA Cancer J Clin 66: 7-30, 2016.

3. Chiang YC, Chen CA, Chiang CJ, Hsu TH, Lin MC, You SL, Cheng WF and Lai MS: Trends in incidence and survival outcome of epithelial ovarian cancer: 30-Year national population-based registry in Taiwan. J Gynecol Oncol 24: 342-351, 2013.

4. Austin RM: Benign to malignant transformation in epithelial ovarian tumors. Hum Pathol 24: 562-563, 1993.

5. Fitzgibbons PL, Henson DE and Hutter RV; Cancer Committee of the College of American Pathologists: Benign breast changes and the risk for subsequent breast cancer: An update of the 1985 consensus statement. Arch Pathol Lab Med 122: 1053-1055, 1998.

6. Dupont WD, Page DL, Parl FF, Vnencak-Jones CL, Plummer WD Jr, Rados MS and Schuyler PA: Long-term risk of breast cancer in women with fibroadenoma. N Engl J Med 331: 10-15, 1994.

7. McDivitt RW, Stevens JA, Lee NC, Wingo PA, Rubin GL and Gersell D; The Cancer and Steroid Hormone Study Group: Histologic types of benign breast disease and the risk for breast cancer. Cancer 69: 1408-1414, 1992.

8. Powell DE, Puls L and van Nagell J Jr: Current concepts in epithelial ovarian tumors: Does benign to malignant transformation occur? Hum Pathol 23: 846-847, 1992.

9. Waldemarson S, Krogh M, Alaiya A, Kirik U, Schedvins K, Auer G, Hansson KM, Ossola R, Aebersold R, Lee H, et al: Protein expression changes in ovarian cancer during the transition from benign to malignant. J Proteome Res 11: 2876-2889, 2012. 
10. de Haan J, Verheecke M and Amant F: Management of ovarian cysts and cancer in pregnancy. Facts Views Vis Obgyn 7: 25-31, 2015.

11. Ponting CP, Oliver PL and Reik W: Evolution and functions of long noncoding RNAs. Cell 136: 629-641, 2009.

12. Cabili MN, Trapnell C, Goff L, Koziol M, Tazon-Vega B, Regev A and Rinn JL: Integrative annotation of human large intergenic noncoding RNAs reveals global properties and specific subclasses. Genes Dev 25: 1915-1927, 2011.

13. Silva JM, Boczek NJ, Berres MW, Ma X and Smith DI: LSINCT5 is over expressed in breast and ovarian cancer and affects cellular proliferation. RNA Biol 8: 496-505, 2011.

14. Medrzycki M, Zhang Y, Zhang W, Cao K, Pan C, Lailler N, McDonald JF, Bouhassira EE and Fan Y: Histone h1.3 suppresses h19 noncoding RNA expression and cell growth of ovarian cancer cells. Cancer Res 74: 6463-6473, 2014.

15. Guan Y, Kuo WL, Stilwell JL, Takano H, Lapuk AV, Fridlyand J, Mao JH, Yu M, Miller MA, Santos JL, et al: Amplification of PVT1 contributes to the pathophysiology of ovarian and breast cancer. Clin Cancer Res 13: 5745-5755, 2007.

16. Qiu JJ, Wang Y, Ding JX, Jin HY, Yang G and Hua KQ: The long non-coding RNA HOTAIR promotes the proliferation of serous ovarian cancer cells through the regulation of cell cycle arrest and apoptosis. Exp Cell Res 333: 238-248, 2015.

17. Gloss B, Moran-Jones K, Lin V, Gonzalez M, Scurry J, Hacker NF, Sutherland RL, Clark SJ and Samimi G: ZNF30OP1 encodes a lincRNA that regulates cell polarity and is epigenetically silenced in type II epithelial ovarian cancer. Mol Cancer 13: 3, 2014

18. Qiu JJ, Lin YY, Ye LC, Ding JX, Feng WW, Jin HY, Zhang Y, Li Q and Hua KQ: Overexpression of long non-coding RNA HOTAIR predicts poor patient prognosis and promotes tumor metastasis in epithelial ovarian cancer. Gynecol Oncol 134: 121-128, 2014

19. Zhou M, Sun Y, Sun Y, Xu W, Zhang Z, Zhao H, Zhong Z and Sun J: Comprehensive analysis of lncRNA expression profiles reveals a novel lncRNA signature to discriminate nonequivalent outcomes in patients with ovarian cancer. Oncotarget 7 : 32433-32448, 2016.

20. Pelusi G, Taroni B and Flamigni C: Benign ovarian tumors. Front Biosci 2: g5-7, 1997.

21. Qiu JJ, Lin YY, Ding JX, Feng WW, Jin HY and Hua KQ: Long non-coding RNA ANRIL predicts poor prognosis and promotes invasion/metastasis in serous ovarian cancer. Int J Oncol 46: $2497-2505,2015$

22. Gao Y, Meng H, Liu S, Hu J, Zhang Y, Jiao T, Liu Y, Ou J, Wang D, Yao L, et al: LncRNA-HOST2 regulates cell biological behaviors in epithelial ovarian cancer through a mechanism involving microRNA let-7b. Hum Mol Genet 24: 841-852, 2015.

23. Wang H, Fu Z, Dai C, Cao J, Liu X, Xu J, Lv M, Gu Y, Zhang J, Hua $\mathrm{X}$, et al: LncRNAs expression profiling in normal ovary, benign ovarian cyst and malignant epithelial ovarian cancer. Sci Rep 6: 38983, 2016.

24. Yang J, Chen L, Kong X, Huang T and Cai YD: Analysis of tumor suppressor genes based on gene ontology and the KEGG pathway. PLoS One 9: e107202, 2014.

25. Zhang EB, Yin DD, Sun M, Kong R, Liu XH, You LH, Han L, Xia R, Wang KM, Yang JS, et al: P53-regulated long non-coding RNA TUG1 affects cell proliferation in human non-small cell lung cancer, partly through epigenetically regulating HOXB7 expression. Cell Death Dis 5: e1243, 2014.

26. Khalil AM, Guttman M, Huarte M, Garber M, Raj A, Rivea Morales D, Thomas K, Presser A, Bernstein BE, van Oudenaarden A, et al: Many human large intergenic noncoding RNAs associate with chromatin-modifying complexes and affect gene expression. Proc Natl Acad Sci USA 106: 11667-11672, 2009.
27. G Hendrickson D, Kelley DR, Tenen D, Bernstein B and Rinn JL: Widespread RNA binding by chromatin-associated proteins. Genome Biol 17: 28, 2016.

28. Salmena L, Poliseno L, Tay Y, Kats L and Pandolfi PP: A ceRNA hypothesis: The Rosetta Stone of a hidden RNA language? Cell 146: 353-358, 2011.

29. Zhou M, Wang X, Shi H, Cheng L, Wang Z, Zhao H, Yang L and Sun J: Characterization of long non-coding RNA-associated ceRNA network to reveal potential prognostic lncRNA biomarkers in human ovarian cancer. Oncotarget 7: 12598-12611, 2016.

30. Cole SW, Nagaraja AS, Lutgendorf SK, Green PA and Sood AK: Sympathetic nervous system regulation of the tumour microenvironment. Nat Rev Cancer 15: 563-572, 2015.

31. Huan HB, Wen XD, Chen XJ, Wu L, Wu LL, Zhang L, Yang DP, Zhang X, Bie P, Qian C, et al: Sympathetic nervous system promotes hepatocarcinogenesis by modulating inflammation through activation of alpha1-adrenergic receptors of Kupffer cells. Brain Behav Immun 59: 118-134, 2017.

32. Magnon C, Hall SJ, Lin J, Xue X, Gerber L, Freedland SJ and Frenette PS: Autonomic nerve development contributes to prostate cancer progression. Science 341: 1236361, 2013.

33. Luo M, Li Z, Wang W, Zeng Y, Liu Z and Qiu J: Long non-coding RNA H19 increases bladder cancer metastasis by associating with EZH2 and inhibiting E-cadherin expression. Cancer Lett 333: 213-221, 2013

34. Hirata H, Hinoda Y, Shahryari V, Deng G, Nakajima K, Tabatabai ZL, Ishii N and Dahiya R: Long noncoding RNA MALAT1 promotes aggressive renal cell carcinoma through Ezh2 and interacts with miR-205. Cancer Res 75: 1322-1331, 2015.

35. Crea F, Watahiki A, Quagliata L, Xue H, Pikor L, Parolia A, Wang Y, Lin D, Lam WL, Farrar WL, et al: Identification of a long non-coding RNA as a novel biomarker and potential therapeutic target for metastatic prostate cancer. Oncotarget 5: 764-774, 2014.

36. Zhou M, Zhong L, Xu W, Sun Y, Zhang Z, Zhao H, Yang L and Sun J: Discovery of potential prognostic long non-coding RNA biomarkers for predicting the risk of tumor recurrence of breast cancer patients. Sci Rep 6: 31038, 2016.

37. Zhou M, Diao Z, Yue X, Chen Y, Zhao H, Cheng L and Sun J: Construction and analysis of dysregulated lncRNA-associated ceRNA network identified novel lncRNA biomarkers for early diagnosis of human pancreatic cancer. Oncotarget 7: 5638356394, 2016.

38. Zhou M, Guo M, He D, Wang X, Cui Y, Yang H, Hao D and Sun J: A potential signature of eight long non-coding RNAs predicts survival in patients with non-small cell lung cancer. J Transl Med 13: 231, 2015.

39. Zhou M, Xu W, Yue X, Zhao H, Wang Z, Shi H, Cheng L and Sun J: Relapse-related long non-coding RNA signature to improve prognosis prediction of lung adenocarcinoma. Oncotarget 7: 29720-29738, 2016.

40. Ma Y, Lu Y and Lu B: MicroRNA and long non-coding RNA in ovarian carcinoma: Translational insights and potential clinical applications. Cancer Invest 34: 465-476, 2016.

41. Yim GW, Kim HJ, Kim LK, Kim SW, Kim S, Nam EJ and Kim YT: Long non-coding RNA HOXA11 antisense promotes cell proliferation and invasion and predicts patient prognosis in serous ovarian cancer. Cancer Res Treat: Oct 11, 2016 (Epub ahead of print). doi: 10.4143/crt.2016.263. 